\title{
Un modèle d'interaction d'une houle non-linéaire avec une marche immergée
}

\author{
Max Belzons \\ Maître de Conférences, IUSTI, UMR-139, Université de Provence \\ 5, rue Enrico Fermi, 13453 Marseille cedex 13 \\ Tél.0491106876 Max.Belzons@polytech.univ-mrs.fr \\ Cyril Dulou \\ ATER, UMR5805 EPOC, Université Bordeaux I \\ Avenue des Facultés, 33405 Talence cedex \\ Tél. 0556848835 c.dulou@epoc.u-bordeaux.fr
}

\begin{abstract}
Résumé
Nous présentons une modélisation potentielle d'une houle bichromatique faiblement non-linéaire résultant de la perturbation d'une onde de Stokes par une onde libre de faible amplitude et de fréquence double. Cette modélisation rend compte du transfert d'énergie vers les basses fréquences, ici vers le fondamental qui est donc perturbé par l'onde libre. Un modèle d'interaction de cette houle avec des obstacles (ressaut ou marche) est ensuite proposé. L'intérêt de ce modèle est de donner accès à l'ensemble du champ hydrodynamique 3D. Il est en bon accord avec l'observation expérimentale de production ou de suppression d'onde libre par des accidents bathymétriques.

We present a potential modelling of a weakly nonlinear bichromatic wave perturbed by a low amplitude free wave having a frequency twice that of the fundamental. This model addresses the energy transfer to the low frequency range, that is, in the present case, to the fundamental which is then perturbed by the free wave. A model of interaction of this wave with bottom obstacles (shelf or sill) is then proposed. The interest of this model is that it gives the whole $3 D$ wavefield. It is in good agreement with the experimental observations of the production or the suppression of free wave by an abruptly changing bathymetry
\end{abstract}

\section{$\underline{\text { 1.Introduction }}$}

La propagation de la houle en zone littorale, en profondeur intermédiaire ou faible, est dominée par des interactions non-linéaires onde-onde. Elles induisent des transferts d'énergie entre les composantes spectrales de la houle incidente, et ceux qui se produisent vers les basses fréquences ont une forte influence sur le transport sédimentaire. Ces interactions se manifestent aussi lorsque la houle est diffractée par des obstacles immergés. Leur modélisation 
expérimentale nécessite en particulier une bonne mâtrise du champ de vagues incident. En fait, lorsque 1'on cherche à produire une vague monochromatique non-linéaire (de fréquence $f$ et de nombre d'onde $k$ ) dans un canal à houle au moyen d'un batteur, ou bien lors de sa propagation en eau de profondeur non constante, il apparaît des modes supplémentaires de faible amplitude, de fréquence $n f(n=1,2, \ldots)$, de nombre d'onde $K_{n} \neq n k$. Ces modes libres se propagent avec leur propre vitesse de phase $c_{n}=2 \pi n f / K_{n}$. Ils sont la conséquence de l'aspect nonlinéaire de la propagation, exprimé ici dans des conditions de raccordement aux parois solides que constituent le batteur ou le fond (Madsen, 1971). Ce phénomène peut être un artefact expérimental conduisant à de mauvaises interprétations lors d'expériences sur le transport sédimentaire ou sur des mesures d'efforts sur des structures. On s'est intéressé ici à une modélisation de ces interactions qui bien que très simplifiée puisse donner accès au champ hydrodynamique et rendre compte, au moins qualitativement, des observations expérimentales de propagation de houle au-dessus d'accidents bathymétriques, et en particulier de production ou de suppression d'onde libre.

\section{Modélisation}

\subsection{Onde faiblement non-linéaire perturbée par une onde libre}

On suppose que l'eau, est incompressible et sans viscosité et que l'écoulement est irrotationel. On considère une houle progressive se propageant selon les $\mathrm{x}$ positifs au-dessus d'un fond horizontal imperméable d'équation $y=-h$. Soit $\eta(x, t)$ l'élongation au temps t et $\varphi(x, y, t)$ le potentiel de vitesse. Les équations à résoudre sont :

$$
\begin{array}{ll}
\Delta \varphi=0, \text { dans tout le fluide } & 1 \mathrm{a}) \\
\varphi_{\mathrm{y}}=0, \text { en } \mathrm{y}=-\mathrm{h} & 1 \mathrm{~b}) \\
\eta_{\mathrm{t}}+\varphi_{\mathrm{x}} \eta_{\mathrm{x}}-\varphi_{\mathrm{y}}=0, \text { en } \mathrm{y}=\eta \quad \text { (condition dynamique) } & 1 \mathrm{c}) \\
\varphi_{\mathrm{t}}+\left[\varphi_{\mathrm{x}}{ }^{2}+\varphi_{\mathrm{y}}{ }^{2}\right] / 2+\mathrm{g} \eta=0, \text { en } \mathrm{y}=\eta \quad \text { (condition cinématique) } & 1 \mathrm{~d})
\end{array}
$$

où g est l'accélération de la pesanteur.

On considère une houle bichromatique, constituée d'une onde principale faiblement non-linéaire de fréquence $f$ et de nombre d'onde $\mathrm{k}$, qui interagit avec une onde libre de fréquence $2 \mathrm{f}$. L'onde principale est d'amplitude suffisamment faible pour se limiter au fondamental d'amplitude $\varepsilon a, \varepsilon=k a<<1$, et au $1^{\text {er }}$ harmonique d'amplitude $\mathrm{O}\left(\varepsilon^{2} a\right)$. L'onde libre a une amplitude $\mathrm{a}_{\mathrm{L}}=\mathrm{O}(\mu \mathrm{a})$, avec $\mu=\mathrm{O}\left(\varepsilon^{2}\right)$. On fait une modélisation en perturbation de cette interaction faiblement non-linéaire par un développement sur les deux petits paramètres $\varepsilon$ et $\mu$ limité aux termes significatifs les plus bas. On a : 


$$
\begin{array}{lcc}
\eta=\varepsilon \eta_{1}+\varepsilon^{2} \eta_{2}+O\left(\varepsilon^{3}\right) & \text { et } & \varphi=\varepsilon \varphi_{1}+\varepsilon^{2} \varphi_{2}+O\left(\varepsilon^{3}\right) \\
\text { avec } & \eta_{1}=\eta_{0}+\mu \eta_{L} & \varphi_{1}=\varphi_{0}+\mu \varphi_{L} \\
\text { et } & \varphi_{2}=\varphi_{02}+\mu \varphi_{L} & \eta_{2}=\eta_{02}+\mu \eta_{L 2}
\end{array}
$$

\subsubsection{Régime linéaire}

Il est décrit au $1^{\text {er }}$ ordre en $\varepsilon$ par :

$$
\begin{array}{rlrl}
\eta_{1} & =\eta_{0}+\mu \eta_{\mathrm{L}} & \text { et } \quad \varphi_{1}=\varphi_{0}+\mu \varphi_{\mathrm{L}} \\
\text { avec } \quad \eta_{0} & =\mathrm{a} \cos (\omega \mathrm{t}-\mathrm{kx}), \quad \omega=2 \pi \mathrm{f} \quad \text { et } \quad \eta_{\mathrm{L}}=\mathrm{a}_{\mathrm{L}} \cos \left(2 \omega \mathrm{t}-\mathrm{k}_{\mathrm{L}} \mathrm{X}+\psi_{\mathrm{L}}\right),
\end{array}
$$

où les couples $(\omega, \mathrm{k})$ et $\left(2 \omega, \mathrm{k}_{\mathrm{L}}\right)$ vérifient la relation de dispersion linéaire.

\subsubsection{Régime faiblement non-linéaire}

Il est décrit au $2^{\text {ème }}$ ordre en $\varepsilon$ en exprimant les conditions 1c) et 1d) non plus sur la surface libre inconnue, mais en $y=0$, par remplacement du potentiel par son expression approchée : $\quad \varphi=\varepsilon \varphi_{1}+\varepsilon^{2}\left(\varphi_{2}+\eta_{1} \varphi_{L y}\right)+O\left(\varepsilon^{3}\right)$ où $\eta_{1}$ est considérée constante. En utilisant alors les relations 2) ce développement prend la forme:

$$
\varphi(x, \eta, t)=\varepsilon\left[\varphi_{0}+\mu \varphi_{L}\right]+\varepsilon^{2}\left[\varphi_{02}+\mu \varphi_{I 2}+\left(\eta_{0}+\mu \eta_{L}\right)\left(\varphi_{0 y}+\mu \varphi_{L y}\right)\right]
$$

à reporter dans $1 \mathrm{c}$ ) et $1 \mathrm{~d}$ ) exprimées en $\mathrm{y}=0$. A l'ordre $\varepsilon$ on retrouve la solution linéaire $\varphi_{0}, \eta_{0}$ et $\varphi_{L}, \eta_{L}$. A l'ordre $\varepsilon^{2}$ on retrouve d'une part la solution de Stokes $\varphi_{02}, \eta_{02}$ et d'autre part la correction non-linéaire $\varphi_{0 \mathrm{~L}}, \eta_{0 \mathrm{~L}}$ due à l'onde libre, par résolution du système d'équations issu de l'identification sur $\mu$ :

$$
\begin{aligned}
& \eta_{L 2 t}-\varphi_{L 2 y}+\varphi_{L x} \eta_{0 x}+\varphi_{0 x} \eta_{L x}-\eta_{0} \varphi_{L y y}-\eta_{L} \varphi_{0 y y}=0 \quad \text { en } y=0 \\
& \varphi_{\mathrm{L} 2 \mathrm{t}}+\mathrm{g} \eta_{\mathrm{L} 2}+\eta_{0} \varphi_{\mathrm{Lyt}}+\eta_{\mathrm{L}} \varphi_{0 \mathrm{yt}}+\varphi_{0 \mathrm{x}} \varphi_{\mathrm{Lx}}+\varphi_{0 \mathrm{y}} \varphi_{\mathrm{Ly}}=0 \text { en } \mathrm{y}=0
\end{aligned}
$$

où les interactions quadratiques exprimées à partir de la solution linéaire, produisent les termes « somme » et « différence », $\left(3 \omega, \mathrm{k}_{\mathrm{L}}+\mathrm{k}\right)$ et $\left(\omega, \mathrm{k}_{\mathrm{L}}-\mathrm{k}\right)$.

Seul le terme «différence » a été par la suite retenu, car dans les expériences on s'intéresse surtout à la perturbation du fondamental et les hautes fréquences $(\geq 4.5$ $\mathrm{Hz}$ pour le terme somme dans les expériences) seraient rapidement atténuées par dissipation visqueuse. L'élongation de l'onde incidente bichromatique, faiblement non-linéaire, est alors:

$\eta=\operatorname{acos}(\omega t-k x)+a_{L 2} \cos \left(\omega t-\left(k_{L}-k\right) x+\Psi_{L}\right)+a_{2} \cos 2(\omega t-k x)+a_{L} \cos \left(2 \omega t-k_{L} x+\Psi_{L}\right)$

où $\mathrm{a}_{2}$ et l'amplitude du $1^{\mathrm{er}}$ harmonique de Stokes et $\mathrm{a}_{\mathrm{L} 2}$ l'amplitude de l'interaction non-linéaire «différence» du fondamental avec l'onde libre. Pour satisfaire l'équation de Laplace et la condition au fond, le potentiel de cette perturbation doit être de la forme:

$\varphi_{\mathrm{L} 2}=\mathrm{A}_{\mathrm{L} 2} \cosh \left[\left(\mathrm{k}_{\mathrm{L}}-\mathrm{k}\right)(\mathrm{h}-\mathrm{y})\right] \sin \left(\omega \mathrm{t}-\left(\mathrm{k}_{\mathrm{L}}-\mathrm{k}\right) \mathrm{x}+\Psi_{\mathrm{L}}\right)$

En reportant les expressions de $\eta_{\mathrm{L} 2}$ et $\varphi_{\mathrm{L} 2}$ dans $3 \mathrm{a}$ ) et $3 b$ ), on obtient les amplitudes: 


$$
\begin{aligned}
& a_{L 2}=\left[A \omega \cosh \left(k_{L}-k\right) h+B\left(k_{L}-k\right) \sinh \left(k_{L}-k\right) h\right] /\left[\omega^{2} \cosh \left(k_{L}-k\right) h-g\left(k_{L}-k\right) \sinh \left(k_{L}-k\right) h\right] \\
& A_{L 2}=[A g+B \omega] /\left[-\omega^{2} \cosh \left(k_{L}-k\right) h+g\left(k_{L}-k\right) \sinh \left(k_{L}-k\right) h\right] \\
& \text { où } \quad A=a a_{L} g\left(k_{L}-k\right)\left(k_{L}+2 k\right) / 4 \omega \quad B=a a_{L}\left(g^{2} k_{L} / 2 \omega^{2}-3 \omega^{2}\right) / 2
\end{aligned}
$$

Le $1^{\text {er }}$ harmonique de Stokes et l'onde libre de même fréquence vont produire un battement de longueur $\mathrm{L}=2 \pi /\left(\mathrm{k}_{\mathrm{L}}-2 \mathrm{k}\right)$ observé expérimentalement dans l'enveloppe du terme de fréquence $2 \mathrm{f}$ de l'onde. Les résultats 4) sont identiques à ceux que Dalzell (1999) a obtenus dans un cadre plus général. Ils décrivent l'élévation de la surface libre par rapport à son niveau moyen : $-a^{2} k / 2 \sinh (2 k h)-a_{L}{ }^{2} k_{L} / 2 \sinh \left(2 k_{L} h\right)$.

\subsection{Propagation d'une onde bichromatique au dessus d'une marche}

A partir de ce modèle en profondeur constante on représente ensuite la propagation au-dessus d'un fond constitué par une simple marche.

\subsubsection{Régime linéaire}

Pour exprimer les conditions de raccordement de part et d'autre d'une discontinuité de profondeur il est indispensable d'ajouter la contribution d'une infinité de modes évanescents à celle de chaque mode propagatif pour obtenir l'expression complète du potentiel (Takano, 1960). Ces modes évanescents, en nombre obligatoirement fini dans la modélisation, sont impliqués dans la conservation du potentiel et du flux à chaque discontinuité, ainsi que dans la condition d'imperméabilité de la paroi verticale. Ces conditions aux limites sont généralement exprimées en tirant avantage des propriétés d'orthogonalité des divers modes du potentiel (Kirby \& Dalrymple, 1983). Dans le cas d'un double ressaut, ou marche, en prenant $n$ modes évanescents pour l'onde principale et $n_{1}<n$ pour l'onde libre, le problème se ramène à la résolution de l'équation matricielle :

$$
\mathrm{MX}_{0}=\mathrm{S}
$$

$X_{0}$ est le vecteur des $2\left(n+n_{1}\right)+8$ coefficients inconnus du potentiel linéaire, $M$ est la matrice de ces coefficients, et $S$ le vecteur des $2\left(n+n_{1}\right)+8$ coefficients connus à partir de la donnée des amplitudes complexes des 2 ondes, et de leur coefficients de réflexion en aval de la marche (Rey, 1991).

\subsubsection{Régime non-linéaire}

Ayant $\mathrm{X}_{0}$ on calcule les interactions non-linéaires. Ces interactions, faibles corrections du régime linéaire, interviennent aussi dans les expressions des conditions limites aux 2 discontinuités, sous la forme d'une faible correction additive et connue $\operatorname{Inter}\left(\mathrm{X}_{0}\right)$ du vecteur $\mathrm{S}$. On obtient ainsi une nouvelle expression matricielle du problème sous la forme :

$$
\mathrm{MX}=\operatorname{Snl}\left(\mathrm{X}_{0}\right)
$$

où $\mathrm{Snl}=\mathrm{S}+\operatorname{Inter}\left(\mathrm{X}_{0}\right)$ est le nouveau vecteur connu. La résolution de 6 ) fournit une valeur approchée des coefficients du potentiel non-linéaire. On procède ensuite par itération jusqu'à obtenir la convergence. 
L'expression exacte du flux d'énergie associé à une onde perturbée exigerait un développement à des ordres plus élevés. On utilise donc une expression approchée du flux en faisant l'addition de la contributions linéarisée de chaque composante de l'onde perturbée. On obtient ainsi les flux incident, réfléchi et transmis et par suite les coefficients de réflexion $\mathrm{R}$ et de transmission $\mathrm{T}$ pour l'énergie.

\section{Comparaison avec les expériences}

Nous avons confronté ce modèle à diverses expériences de propagation de vagues en régime faiblement non-linéaire, soit pour un nombre de Ursell $\mathrm{U}=\mathrm{ak}^{-2} \mathrm{~h}^{-3}<<1$.

\subsection{Propagation au dessus d'une marche}

Ces expériences concernaient la propagation d'une vague incidente monochromatique faiblement non linéaire au dessus d'une marche de $2,5 \mathrm{~cm}$ de hauteur, de 4,5 cm de longueur, immergée dans $4 \mathrm{~cm}$ d'eau (Rey et all., 1992). La fig.1a) représente l'enveloppe du fondamental et celle du $1^{\mathrm{er}}$ harmonique de l'onde en fonction de l'abscisse $\mathrm{x}$ le long $\mathrm{du}$ fond. Le bord amont de la barre est (comme dans le modèle) en $x=0$, la fréquence du fondamental est $2,2 \mathrm{~Hz}$, les 3 amplitudes utilisées sont $\mathrm{a}=0,4(\mathrm{O}), 0,9(\Delta)$ et $1,4 \mathrm{~mm}(\square)$, les coefficients de réflexion aval sont de 0,04 pour le fondamental et nul pour le $1^{\text {er }}$ harmonique. Les graphes ont été tracés en normalisant 1'amplitude incidente et l'ordonnée est ainsi en unité arbitraire. Un transfert d'énergie du fondamental vers le $1^{\text {er }}$ harmonique se manifeste pour $\mathrm{a}=0,9$ et surtout pour $\mathrm{a}=1,4 \mathrm{~mm}$, et on constate une modulation anormale du $1^{\text {er }}$ harmonique pour ces 2 amplitudes. Le comportement linéaire n'est observé que pour $\mathrm{a}=0,4 \mathrm{~mm}$.

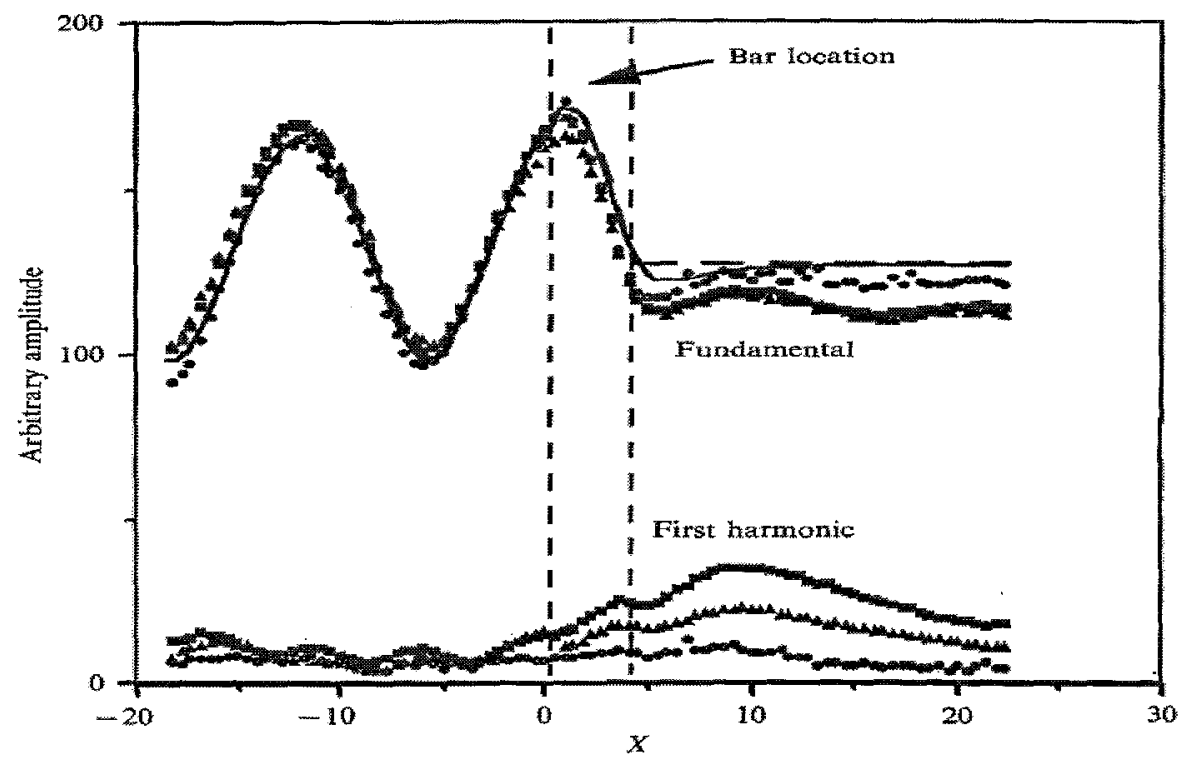

Fig 1a) Evolution spatiale des amplitudes du fondamental et $\mathrm{du} 1^{\mathrm{er}}$ harmonique de l'onde au dessus de la marche: expériences.

Envelope of the fundamental and $I^{\text {rst }}$ harmonic wave above the bar: experiment. 
Les résultats numériques du modèle ont été obtenus dans les mêmes conditions, avec $n=10$ et $n_{1}=3$ (cette valeur de $n_{1}$ s'avère suffisante en raison de la diminution du rôle des modes évanescents lorsque la fréquence croît). On a $U<$ 0,083 pour l'amplitude $\mathrm{a}=0,4 \mathrm{~mm}, \mathrm{U}<0,186$ pour $\mathrm{a}=0,9 \mathrm{~mm}$ et $\mathrm{U}<0,29$ pour a $=1,4 \mathrm{~mm}$. Ils sont en bon accord avec l'expérience, en particulier pour ce qui concerne la modulation du $1^{\mathrm{er}}$ harmonique identifié au battement entre le $1^{\mathrm{er}}$ Stokes et l'onde libre produite au passage de la marche. La production d'onde libre croît en $\mathrm{a}^{2}$, son amplitude dans la région aval est respectivement de 0,025 , 0,12 , et $0,28 \mathrm{~mm}$. La fig. $1 \mathrm{~b}$ ) donne les graphes pour $\mathrm{a}=0,4$ et $\mathrm{a}=1,4 \mathrm{~mm}$.

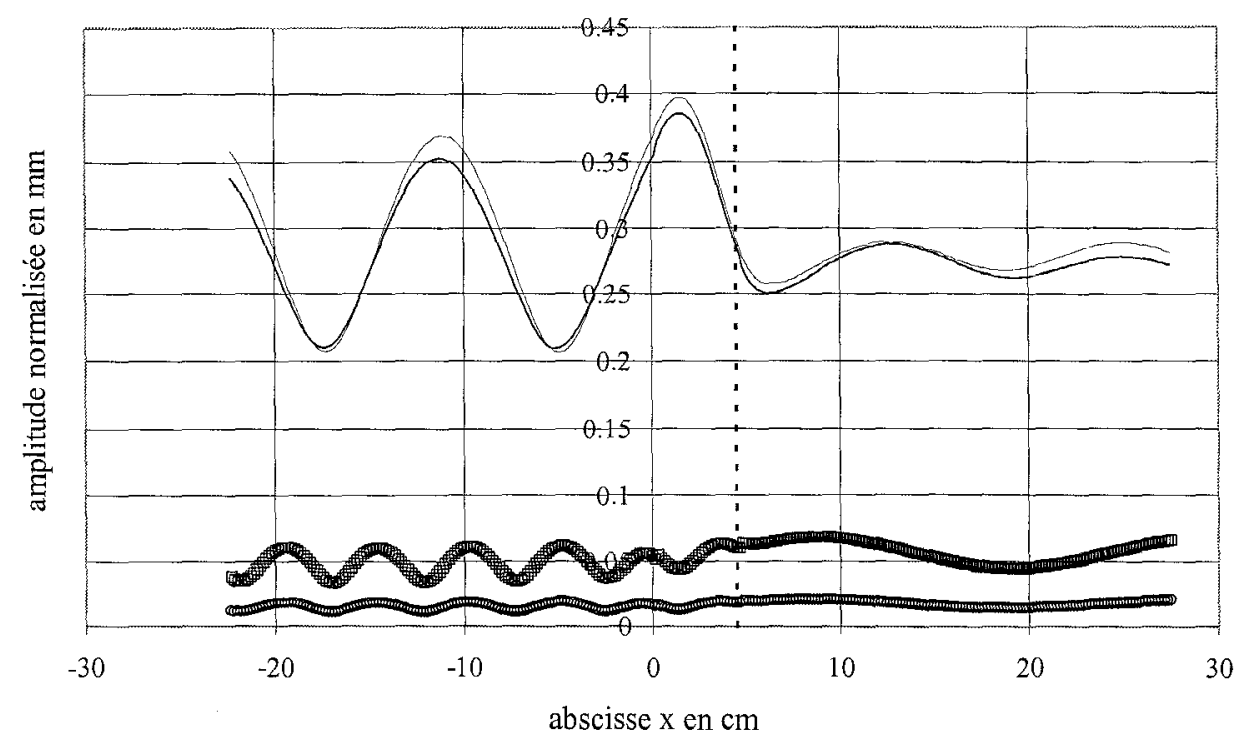

Fig 1b) Evolution spatiale des amplitudes du fondamental et $d u 1^{\text {er }}$ harmonique de l'onde au-dessus de la marche: modélisation.

Envelope of the fundamental and $1^{\text {rst }}$ harmonic wave above the bar: model.

\subsection{Atténuation d'une onde libre parasite}

\subsubsection{Par deshoaling}

Cette méthode consiste à générer la vague perturbée par une onde libre en profondeur $h_{1}$, puis à la faire se propager en aval en profondeur $h_{2}>h_{1}$, par passage au-dessus d'un ressaut situé à un nœud de battement. Cette méthode donne de bons résultats tout au moins pour de faibles taux d'onde libre (Dulou, 2000). On a modélisé ici ces expériences soit avec $\mathrm{f}=1,5 \mathrm{~Hz}, \mathrm{~h}_{1}=6 \mathrm{~cm}, \mathrm{~h}_{2}=8$ $\mathrm{cm}, \mathrm{a}=1 \mathrm{~cm}, \mathrm{a}_{\mathrm{L}}=0,1 \mathrm{~cm}$, et sans aucune réflexion aval. La figure 2 représente les résultats obtenus pour l'amplitude du fondamental et $\mathrm{du} 1^{\mathrm{er}}$ harmonique en fonction de la distance $\mathrm{x}$ au ressaut. Dans le cas où le ressaut est placé à un ventre de battement dans la région amont, on constate en aval un très fort battement du $1^{\text {er }}$ harmonique (x) ainsi q'une importante modulation du fondamental (en trait fin). On a dans ce cas une amplitude d'onde libre en aval de $0,173 \mathrm{~cm}$, nettement supérieure à l'amplitude incidente, et très proche de celle du $1^{\mathrm{er}}$ harmonique de 
Stokes. Dans le cas où le ressaut est placé à un nœud de battement de la région amont, on constate une forte diminution du battement aval du $1^{\text {er }}$ harmonique $(\mathrm{O})$, et de la modulation aval du fondamental (en trait épais). En effet, l'amplitude de l'onde libre transmise n'est plus que de $0,027 \mathrm{~cm}$. Dans le premier cas, l'onde libre produite lors du deshoaling se renforce par interférence constructive avec l'onde incidente, alors que dans le deuxième cas on est en situation favorable d'interférence destructive. On est forcément limité par l'amplitude de l'onde libre qu'un tel ressaut peut produire.

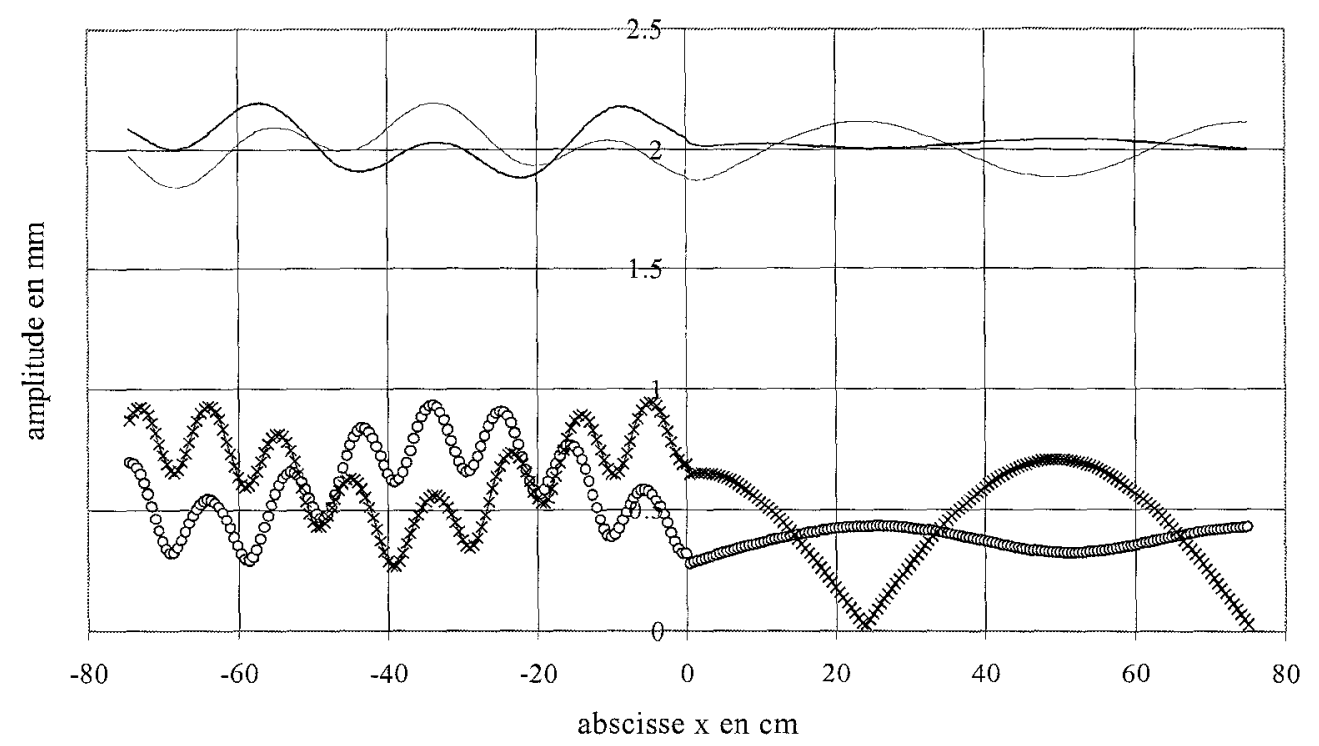

Figure 2 Atténuation ou production d'onde libre par passage sur un ressaut.

Damping or enhancement of a free wave over a shelf.

\subsubsection{Par franchissement d'un seuil}

Une autre méthode d'atténuation d'onde libre consiste à faire se propager l'onde perturbée au dessus d'un seuil en plaçant le bord aval à un nœud de battement. La figure 3 donne les résultats de la simulation pour une onde de fréquence $1,5 \mathrm{~Hz}$, d'amplitudes a $=1 \mathrm{~cm}$ et $\mathrm{a}_{\mathrm{L}}=0,1 \mathrm{~cm}$, de phase $\Psi_{\mathrm{L}}=3,2$, et en absence de réflexion aval. En eau de profondeur constante $h=8 \mathrm{~cm}$ on constate une forte modulation du fondamental (trait fin) associée à un fort battement $(\mathrm{x})$. En présence d'une marche d'épaisseur $1,5 \mathrm{~cm}$ et de longueur $15 \mathrm{~cm}$, placée sur le fond avec son bord aval (trait pointillé) à un nœud de battement, on constate que l'amplitude de l'onde libre dans la région aval est réduite à $a_{L}=0,04 \mathrm{~cm}$, soit nettement inférieure à l'amplitude $\mathrm{a}_{2}=0,179 \mathrm{~cm}$ du $1^{\mathrm{er}}$ harmonique de Stokes. Il en résulte dans la région aval, une forte diminution du battement $(\mathrm{O})$ et de la modulation du fondamental (trait épais). Dans les expériences de Dulou (2000) le même résultat était cependant atteint avec une épaisseur de marche de seulement $0,5 \mathrm{~cm}$. 


\section{Conclusions}

On a modélisé la perturbation d'une onde faiblement non-linéaire par une onde libre de faible amplitude et de fréquence double de celle du fondamental, ainsi que son interaction avec des accidents bathymétriques. Ce modèle a pour principal avantage de donner accès à tout le champ hydrodynamique 3D. Il est cependant

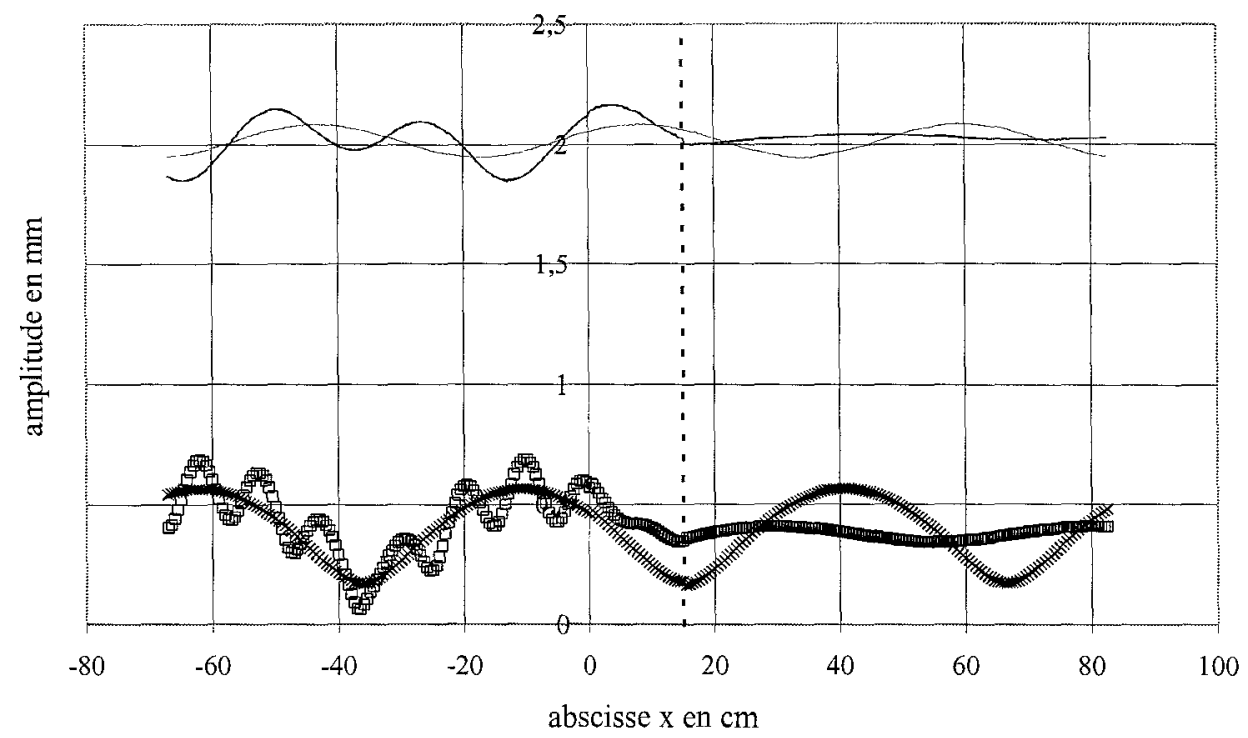

Figure 3 filtrage d'onde libre par passage sur un seuil.

Filtering of free wave by passing over a sill.

limité à de faibles non linéarités, $\varepsilon$ et $\mu<<1$, et, comme en théorie de Stokes à des profondeurs intermédiaires telles que $0,25<\mathrm{kh}<3.14$. Il est aussi pénalisé par l'absence de dissipation et de vorticité. Le modèle est cependant en bon accord qualitatif avec les observations de production ou d'atténuation d'une onde libre par des ressauts ou des seuils, où les modes évanescents jouent un rôle prépondérant, surtout à basse fréquence (Rey, 1991). Il pourrait servir de base à un modèle de propagation 3D au-dessus de fonds cylindriques quelconques par discrétisation du profil en une série de marches, et aussi être étendu au cas plus fortement non-linéaire par un développement à des ordres supérieurs en $\varepsilon$ et $\mu$.

\section{Bibliographie}

Dalzell, J.F., 1999, A note on finite depth second-order wave-wave interactions. Applied Ocean Research, 21, 105-111.

Dulou, C., 2000, Interactions houle-sédiments: application à la formation des barres littorales, thèse, Université de Provence.

Kirby, J. T. \& Dalrymple, R. A., 1983, Propagation of obliquely incident water waves over a trench. J. Fluid Mech. 133, 47-63. 
Madsen, O. L., 1971, On the generation of long waves. J. Geophysical Research $76, \mathrm{~N}^{\circ} 36,8672-8683$.

Rey, V., 1991, Propagation and local behavior of normally incident gravity waves over varying topography. Eur. J. Mech. B :Fluids, 11(2), 213-232.

Rey, V., Belzons, M. \& Guazzelli, E., 1992, Propagation of surface gravity waves over a rectangular submerged bar. J. Fluid Mech. 235, 453-470.

Takano, K., 1960, Effets d'un obstacle parallélépipédique sur la propagation de la houle. La houille Blanche 15, 247-267. 